\title{
COVID-19 MASS MEDIA INFODEMIC IN SIX EUROPEAN GOUNTRIES
}

\author{
Kejo Starosta ${ }^{1}$, Cristian Bogdan Onete ${ }^{2}$, Raluca Grosu ${ }^{3}$ and Doru Plesea ${ }^{4}$ \\ 1) 2) 3) 4) The Bucharest University of Economic Studies \\ Email: k@ksfx.de; Email: bogdan.onete@com.ase.ro; \\ Email: raluca.petrescu@com.ase.ro; Email: pleseadoru@gmail.com
}

\begin{abstract}
Our paper tackles the development of media reporting during the COVID-19 pandemic, focusing on the January - November 2020 time span, in France, Germany, Romania, Spain, Switzerland, and the United Kingdom. We aim to make media reporting transparent on two dimensions: the coverage of COVID-19-related topics and the negativity of the COVID-19 media reporting. To achieve this goal, we analysed a large news dataset with 841,415 pieces of news - including 202,608 COVID-19 media reports - on an LSTM neural network. The news sentiment data and the corresponding coverage are set in relation to the WHO data on COVID-19 and to Google Trends. This compares the reality, that is WHO data, the perceived and reported reality, that is news data, and the actions based on the perceived and the actual reality, that is Google Trends. The results show that media reporting on COVID19 is unprecedented in terms of coverage and negativity. Furthermore, the study quantifies how far media reporting detached from the facts after the first wave of COVID-19 and how an Infodemic spread across Europe.
\end{abstract}

\section{Keywords}

COVID-19; Pandemic; Infodemic; Media; Online Media; Europe; Sentiment Analysis

\section{JEL Classification}

D83-Search, Learning, Information and Knowledge, Communication, Belief, Unawareness

C45-Neural Networks and Related Topics

O52-Europe 


\section{Introduction}

In these times, nations worldwide are facing important challenges. The COVID-19 virus that spread throughout the world since the beginning of 2020 brought disruptive changes at many levels, such as demographic, economic, or social. Furthermore, from an individual perspective, the current COVID-19- related context had and still has a considerable impact on the daily life of most people around the world. The impact is vast, mainly deriving from the measures aimed at reducing the spreading of the virus. All measures, ranging from the "soft" ones such as working from home, to the "hard" ones-as lockdowns-affect humans in different ways. Somehow, COVID-19 took over our lives. Of course, this is not the first pandemic that affected the world, and for sure, this will not be the last, either. However, only considering the last decades, this is the most important juncture.

The International Monetary Fund classifies the COVID-19 pandemic and the resultant economic crisis as: 'a crisis like no other', 'rare disaster', 'worst economic downturn since the great depression', 'far worse than the global financial crisis', and 'more severe economic fallout than anticipated' (Gopinath, 2020; International Monetary Fund, 2020). Gills (2020) also outlines how this disaster might permanently change the world that we know today. These vivid examples already show how big the impact is for the whole human civilization. This is also reflected in the online media with an unprecedented amount of news reporting on all facets of the COVID-19 pandemic.

Considering news coverage as "the most important information source for the public", especially when acknowledging that "epidemics far exceed the knowledge scope of most ordinary people" (Schwitzer et al., 2005; Chen, Huang and Li, 2020), we investigate the COVID-19-related media reporting focusing on six European countries: France, Germany, Romania, Spain, Switzerland, and the United Kingdom (UK). As the media is the key source of information for the public, this is also the key communication instrument for governments, administrations, policymakers, medical doctors and virus researchers to communicate in this case how the disease could be countered. Thus, it is of key interest to make transparent how the media acted during the pandemic. This is especially true for Europe and the Schengen area as the virus was able to spread more easily due to the absence of borders and the fact that the measures taken against the virus were partly coordinated within the European Union (EU) and the Schengen area.

Thus, we aim to analyse the differences and similarities in the media reporting across the countries under observation and to show how people reacted based on this reporting. In order to achieve our objective, we developed this paper by combining a profound sentiment analysis with a correlation analysis on several variables. In this study, two dimensions reflect the media reporting: the coverage of COVID-19 and the negativity of COVID-19related news. The media reporting - the perceived reality - is set in relation to the actual number COVID-19 cases and the actual number of deaths related to COVID-19 - the actual reality. Further, these variables are set in relation to the Google Trend searches on COVID19. This gives a comprehensive picture of the Infodemic in the countries under observation and how people reacted to it.

\section{Literature Review}

An increasing body of literature on sentiment analysis during the Covid-19 pandemic is available in the scientific community. We put forward some of the recent trajectories encountered in the scientific literature published since the beginning of the pandemic. Considering the main objective of the paper, the literature review centres around two main areas: the use of sentiment analysis for online mass media in general and, in a more specific case, during the COVID-19 pandemic.

The way in which sentiment analysis emerged in general during the last years is thoroughly reviewed and assessed in the papers of Agarwal, Kumar, and Goel (2019) and Zhang et al. 
(2018). At first, some studies influenced the general methodology of this research, especially Förschler and Alfanos' (2017) analysis of online financial news, in which they showed that the numbers of incoming orders and other leading indicators were strongly influenced by media sentiments. Further, Shapiro et al. (2017) demonstrate an outstanding proprietary system for creating sentiment indices, based on online news, that are able to forecast and nowcast several business cycle indicators. Besides their influence on our methodology, both studies also show how the facts in the data and the media reporting correlate over time for their case studies and thus provide important information for the interpretation of the results of this research.

Some research papers focus on the sentiment development during the COVID-19 pandemic. Zhou et al. (2020) analyse the Twitter messages in Australia and especially in local areas of New South Wales. They found that the overall sentiments were decreasing depending on the policies to tackle the pandemic and events related to it. In contrast, Barkur, Vibha and Kamath (2020) showed that for India, the Twitter reactions to the COVID-19 lockdown were mainly positive. Zhu et al. (2020) show how microblogging data can be utilized to gather information about public opinions and ideas to provide decision support for macrocontrol response strategies, measures and risk communication. Further, some studies also address the differences and similarities in Twitter sentiments. In a comprehensive study, Imran et al. (2020) present the Twitter sentiments on COVID-19 events and measures for many countries. Kruspe et al. (2020) did a similar study on European countries and analysed the sentiment situation on Twitter over time with respect to COVID-19 events and announcements. For instance, they showed the deteriorating sentiments after the lockdown announcements across all countries. The most recent study considered in this literature review is the influential one by Cinelli et al. (2020) who analyse the global Infodemic on a range of social media platforms: Twitter, Instagram, YouTube, Reddit and Gab.

Almost all sentiment studies on the COVID-19 pandemic focus on the data of Twitter or social media as they are easier to retrieve. Only very few papers focus on the reporting in the mass media, which is more important as it is the opinion former par excellence. The studies of McCombs and Shaw (1972), Scheufele and Tewksbury (2007), Tan and Weaver (2007), Maurer (2017), Birkland (2018), Guo (2019), and Sciarini and Tresch (2019) among many others in this research area - provide good insights into how agenda setting of the mass media influences public discussions. Tan and Weaver (2007), Birkland (2018), and Sciarini and Tresch (2019) were even able to show how agenda setting in the media directly drove parliamentary discussions and political agenda. It is likely, therefore, that the COVID19 measures are also not solely driven by facts of researchers but by the agenda of the (online) media as well. Buckman et al. (2020) analyse the sentiments of economic news in the United States (US) during the start of the COVID-19 pandemic in 16 major US newspapers. Aslam (2020) developed one of the rare analyses of mass media reporting during the COVID-19 pandemic. Their study revealed that around 52\% of the news headlines in global English news evoked negative sentiments and only 30\% evoked positive sentiments while $18 \%$ were neutral. In addition, Starosta, Budz and Krutwig (2019a) provide a study that analyses the online media coverage and the sentiments in the UK and Germany prior to the COVID-19 pandemic and they show the differences and similarities in the media reporting in Germany and the UK. A vivid example of how the media influences the action of society is the study by Starosta, Budz and Krutwig (2019b) who show how tourist arrivals in many popular tourist destinations are strongly influenced by media reporting in the countries of the travellers.

Based on our knowledge, a cross-country analysis of online mass media sentiments on the COVID-19 pandemic does not exist. It seems that viewing the impact of COVID-19 through the lens of online media reporting, based on sentiments and correlation analyses represents a gap in scientific literature. 


\section{Research Questions}

In order to fill the identified research gap and to get a comprehensive view on the reporting of the online mass media in Europe, we address the following research questions during the course of our research:

- How did the media coverage on COVID-19-related topics evolve in comparison to the actual COVID-19 cases and COVID-19 deaths?

- How did the media negativity (valence) on COVID-19-related topics evolve in comparison to the actual COVID-19 cases and COVID-19 deaths?

- How did the society react (as reflected in the Google Trends) to the media reporting (the perceived reality) and the COVID-19 cases and deaths (the actual facts)?

Aiming to properly answer these questions, we further tackle the topic starting from an empirical premise: the higher the COVID-19 deaths and cases, the higher the media negativity of COVID-19-related news and the larger the media coverage. In line with this premise, lower numbers of COVID-19 cases and deaths should lead to a relief in both: the media should be more optimistic translating in lower negativity and its interest on COVID19-related topics should shrink as reflected in lower coverage.

\section{Methodological Aspects}

In this paper, we investigate the media coverage and its negativity as well as the similarities and differences in COVID-19-related media reporting in a quantitative analysis involving six European countries. The methodology consists of four steps, thoroughly detailed in the next sub-sections: 1) information retrieval from online news; 2) sentiment analysis; 3) creation of sentiment and news coverage time series; 4) correlation and regression analyses.

\section{Data Sources and Information Retrieval}

The main variables approached in our analyses refer to: media coverage of COVID-19, media negativity in COVID-19 reporting, actual COVID-19 cases and deaths and Google Trends on COVID-19 searches.

To analyse media sentiments, we sourced news reports from publicly available news streams in France, Germany, Romania, Spain, Switzerland, and the UK focusing on the January - November 2020 time span. The news streams outlined a comprehensive approach, covering various topics similar to the daily press. An accurate dimension of the available news in the news streams is presented in Table 1.

Table 1. Data Sources

\begin{tabular}{|l|l|l|l|l|l|l|}
\hline & CH & DE & ES & FR & RO & UK \\
\hline Total News Reports & 27,522 & 177,213 & 115,113 & 33,864 & 33,256 & 454,447 \\
\hline $\begin{array}{l}\text { Covid-19 News } \\
\text { Reports }\end{array}$ & 2,297 & 41,352 & 30,509 & 6,948 & 10,021 & 111,481 \\
\hline $\begin{array}{l}\text { Share of Covid-19 } \\
\text { News }\end{array}$ & 0.0834 & 0.2334 & 0.2650 & 0.2051 & 0.3013 & 0.2453 \\
\hline
\end{tabular}

Source: this study

This data is set in relation to the actual COVID-19 cases and deaths as reported by the World Health Organization (WHO, 2020) in the same time span. As an additional data source, we used data from Google Trends (2020) on COVID-19-related searches for each country. 


\section{Sentiment Analysis}

The sentiment analysis specific to each news item in the news corpus under study was carried out by using a supervised learning algorithm, that is, a Long Short-Term Memory (LSTM) artificial neural network, as described in detail by Starosta et al. (2019). To achieve state-of-the-art accuracy, it was necessary for the LSTM network to learn the characteristics of positive and negative news for all five languages under observation: English, French, German, Romanian and Spanish. Thus, we used a gold standard training corpus consisting of 3,000 positive news items and as many negative news items in English derived from Starosta et al. (2019).

To create language models for languages beyond English, all news reports in the gold standard corpus were translated by first class translators into French, German, Romanian and Spanish. The advantage of this approach is that all these models use the same data but in different languages. Therefore, no biases in the language models are created due to the use of different training articles.

We further focus on two major steps of the sentiment analysis pipeline: vectorization of the input and training data and the LSTM network itself. To generate machine-readable text that can be utilized by machine learning algorithms, vectorization of the words and n-grams of text is necessary. As proposed in Mikolov et al. (2013), we vectorized our data with the help of the Word2Vec model. For sentiment analysis, we used a standard LSTM neural network. This is a recurrent neural network that can take sequential data as input data. For example, considering the case of our study, the sequential data is the text of the media reports.

The main advantage of the LSTM sentiment analysis is that it can capture long-term dependencies in sentences and documents. This provides a deeper textual understanding of the sentiment analysis compared to simpler networks or vocabulary-based systems. An LSTM cell can selectively choose what it should remember and what it can forget. For instance, it is possible for the LSTM to take the previous weight states unchanged in the occurrence of a word that carries no sentiment information. This might happen if the current word is just a filler word or a stop word. This is how the long-term memory is developed. With the forget gate, the LSTM can also decide to forget the previous state. Furthermore, the LSTM is able to combine both the previous and the current state and interpolate a new one.

Figure 1 illustrates principles of a standard LSTM cell and shows all the gates for which weights are adjusted during the training and learning process. 


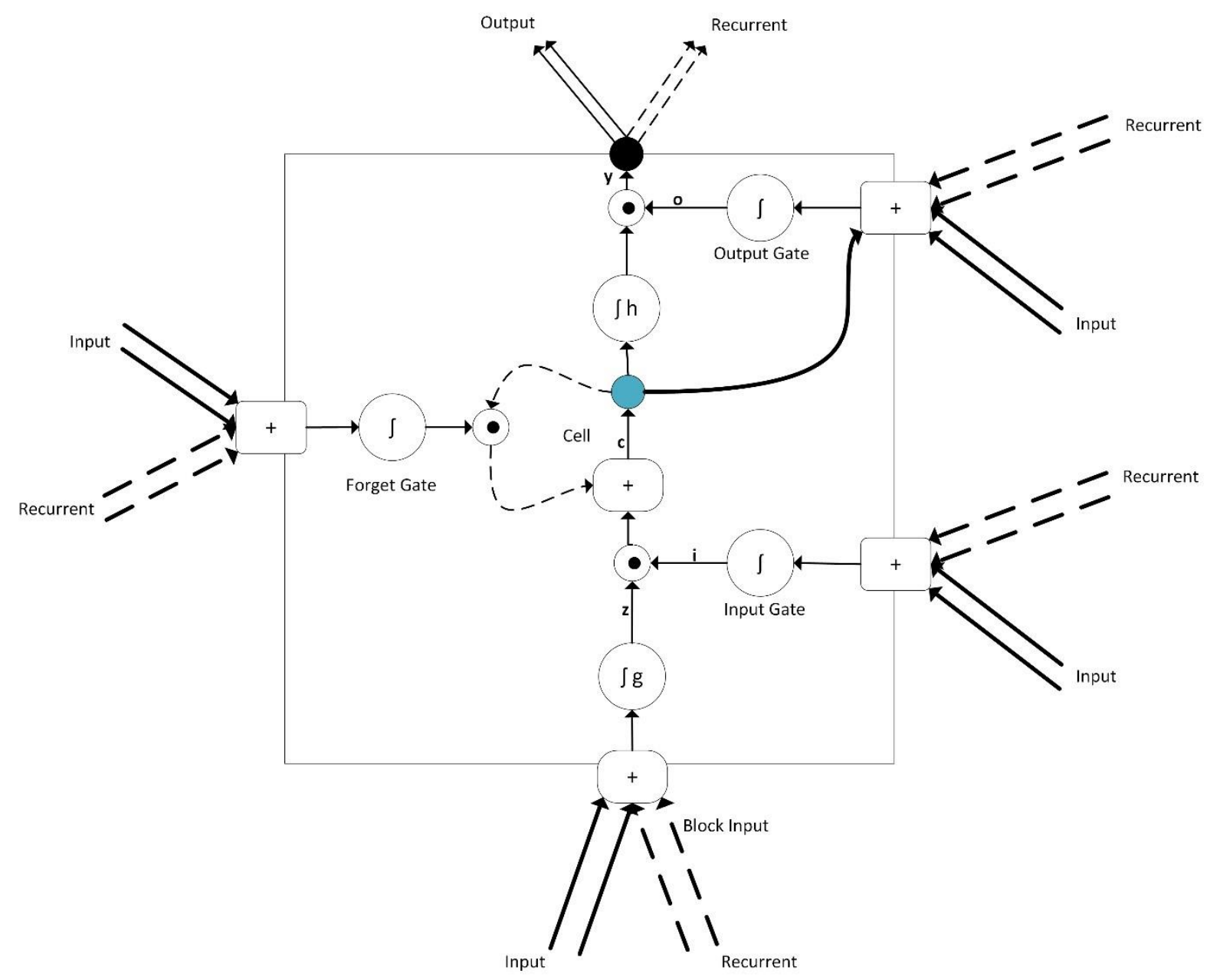

$\longrightarrow$ Unweighted connection

$\longrightarrow$ Weighted connection

$---\rightarrow$ Recurrent connection with time lag

$\int$ Gate activation function (Sigmoid)

fg Input activation function (Tanh)

Ih Output activation function (Tanh)

- Multiplication

$+\quad$ Summation

Figure 1. Principles of a Standard LSTM Cell 
The following paragraphs show which weights can be updated and which parameters these updates depend on.

First, the network computes the information for the input gate $i_{t}$, which defines how much information can pass the input gate (see Equation (1)). How much information can pass the input gate is defined by the input weight matrices $W_{i}$ and $U_{i}$, the current input $x_{t}$, the recurrent input $y_{t-1}$, and the input bias vector $b_{i}$.

$i_{t}=\sigma\left(W_{i} x_{t}+U_{i} y_{t-1}+b_{i}\right)$

What information should pass the cell input gate is defined by the memory cell input $z_{t}$ (see Equation (2)) which depends on the cell input weight matrices $W_{c}$ and $U_{c}$, the current input $x_{t}$, the recurrent input $y_{t-1}$, and the cell input bias vector $b_{c}$.

$z_{t}=\tanh \left(W_{c} x_{t}+U_{c} y_{t-1}+b_{c}\right)$

Apart from computing how much information from the input gate should be used to update the network, another question is how much of the current cell state can be discarded or forgotten because it carries no information for the investigated issue. The forget gate defines how much information can be discarded (see Equation (3)). This is again influenced by the forget input weight matrices $W_{f}$ and $U_{f}$, the current input $x_{t}$, the recurrent input $y_{t-1}$, and the forget input bias vector $b_{f}$.

$f_{t}=\sigma\left(W_{f} x_{t}+U_{f} y_{t-1}+b_{f}\right)$

With the above parameters, the LSTM network can calculate the new cell state $c_{t}$, based on Equation (4).

$c_{t}=i_{t} * z_{t}+f_{t} * c_{t-1}$

How much information can pass the output gate is defined by the output weight matrices $W_{o}, U_{o}$, and $V_{o}$, the current input $x_{t}$, the recurrent input $y_{t-1}$, the current cell state $c_{t}$, and the input bias vector $b_{o}$ (see Equation (5)).

$o_{t}=\sigma\left(W_{o} x_{t}+U_{o} y_{t-1}+V_{0} c_{t}+b_{o}\right)$

Finally, the new output $y_{t}$ is defined by Equation (6).

$y_{t}=o_{t} * \tanh \left(c_{t}\right)$

After determining the weight matrices for all neurons with the help of the gold standard training corpus and creating the language models for all languages, any news article can be classified with the LSTM network.

Figure 2 shows the network architecture unrolled over all time steps (words). The LSTM takes an input vector of 300 dimensions which is the word vector created by the Word2Vec algorithm. The LSTM step reduces the dimensions to 256, which are passed to a recurrent neural network that calculates the output on the two output gates - one for each category. The maximum number of time steps (words) used in this study is 1,000. News articles longer than 1,000 words were truncated. We derived this architecture from Patterson and Gibson (2017), Martin et al. (2018) and Starosta et al. (2019) and, based on our assessment methodology, we were able to confirm its performance. 


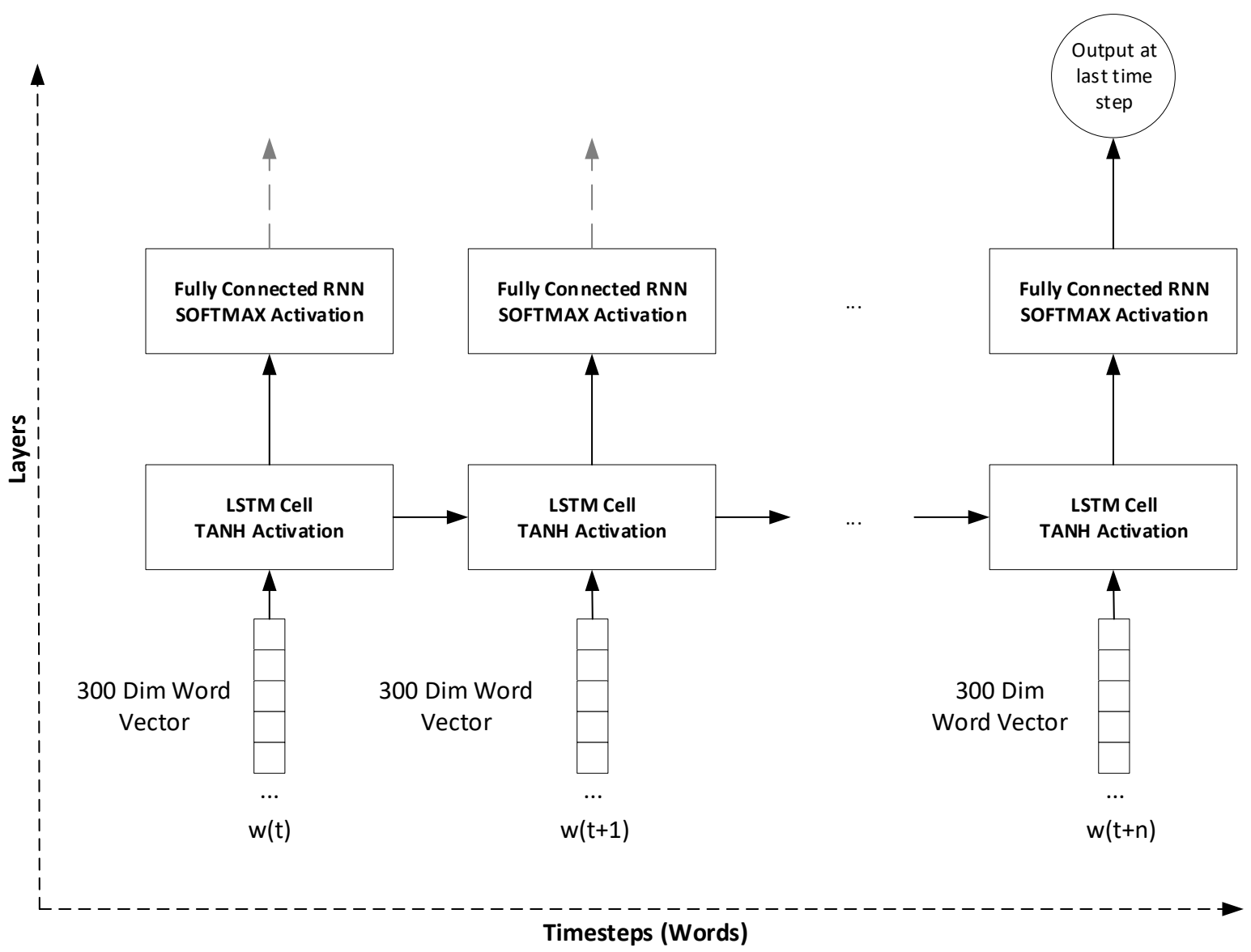

Figure 2. Network Architecture

We assessed the algorithm based on a six-fold cross-validation. For the cross-validation we split up the gold standard training corpus of 3,000 articles into six chunks of 500 articles. We then used five chunks to train the network and the remaining chunk to test the trained network. In a repetitive way, this procedure was employed until all the chunks were tested.

The aggregated results for all languages-English, French, German, Romanian and Spanish-showed that this LSTM architecture led to $88.3 \%$ accurate classifications. In addition, we checked the dispersion of the errors across all confusion matrices of all validation runs in all languages. Also, this test showed that the confusion matrices across all languages and all test chunks were similar without significant outliers. Still, as usual, with these kinds of machine learning procedure, the out-sample performance needs to be continuously monitored by sampling inspections and probably refinements.

\section{News Negativity}

The negativity time series simply measures the negativity (fraction of negative news) of the news over all periods under observation. Its calculus is based on Equation (7). The advantage of having sentiment indices over time is that these indices can be directly compared to the other indicators.

$$
\text { Negativity }_{t}=\left(\frac{N_{t}^{\text {negative }}}{N_{t}^{\text {positive }}+N_{t}^{\text {negative }}}\right)
$$




\section{Correlation Analysis}

To carry out the correlation analysis, we used the ordinary least squares (OLS) estimator, as displayed in Equation (8).

$y=\beta_{0}+I d x_{t} \beta_{1}+\varepsilon$

Where $I d x_{t}$ is the index data of Equation (7).

However, as there were heteroscedasticity and autocorrelations in our time series and creating different models for different countries did not represent a feasible approach, we used Newey-West standard errors to address the OLS estimator-related issues. All correlation analyses were conducted on the first differences and on stationary series.

\section{Results}

The descriptive statistics from the data sources (Table 1) show that the media reporting on the COVID-19 pandemic was unprecedented in the past. A comparison to the studies of Onete et al. (2018) and Starosta, Budz and Krutwig (2019a) that analysed the sentiments and the coverage of the most important topics between 2009 and 2019 showed that there was no topic that could reach either the coverage, or the negativity of the COVID-19 media reporting. This is also in line with Aslam et al. (2020) findings that emphasize high emotional scores with negative polarity of over a half of the 141,208 analysed headlines on COVID-19 of global English news sources.

We further approach the results of our study from an individual perspective, focusing on each analysed country, afterwards comparing the results across countries.

Figure 3 gives an overview of the main analysis of this study for the case of Germany. It shows the share of COVID-19 news on all news (left y-axis) in relation to COVID-19 cases, COVID-19 deaths, Google Trends and the news negativity (right y-axis). 


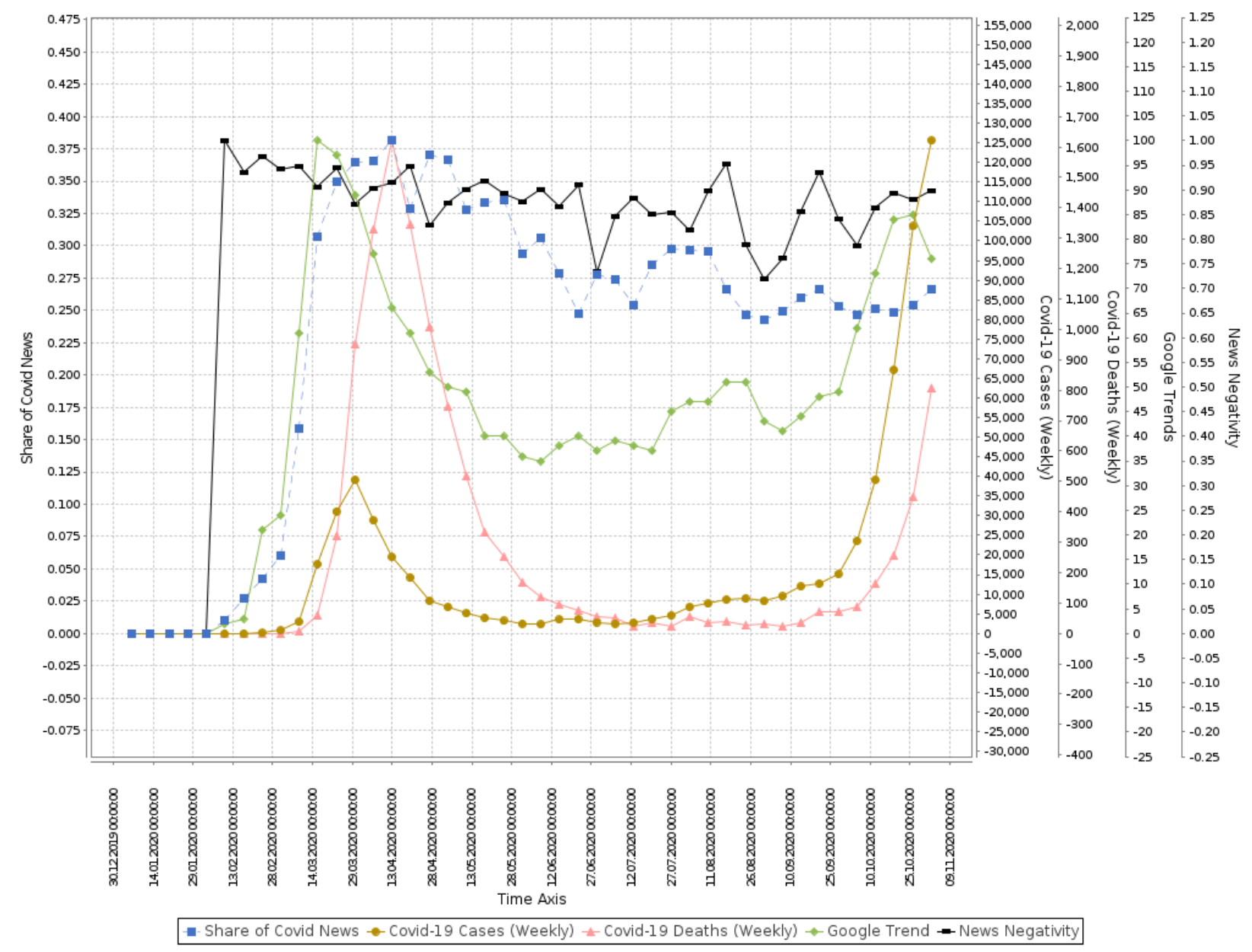

Figure 3. Covid-19 Reporting in Relation to Covid-19 Data and Google Trends for Germany

As the figure shows, the media coverage of COVID-19 news and the news negativity did barely react to the actual figures associated with the COVID-19 pandemic, especially in terms of cases and deaths. Also between May and September, when the number of weekly cases and deaths were very low, the media kept reporting with an unprecedented coverage of COVID-19. The lowest coverage - during the summer months after the first COVID-19 wave - in the week of 22.06.2020 still had a share of 24.6\% of COVID-19-related news. While this was a low point in the COVID-19 pandemic, it was still a number unreached by any other topic before. This leads to the finding that the media coverage and its negativity barely correlate with the COVID-19 cases or COVID-19 deaths (Compare with Table 2). This creates a gap between the perceived reality (created by the media) and the factual reality outlined in the WHO data on COVID-19 cases and deaths. This detachment of the media reporting from the actual development in the reality characterizes an Infodemic - an excessive overreporting detached from available data. This directly leads to the finding how people react to the media and the facts. As expected and analysed by many researchers (McCombs and Shaw, 1972; Scheufele and Tewksbury, 2007; Tan and Weaver, 2007; Maurer, 2017; Birkland, 2018; Guo, 2019; Sciarini and Tresch, 2019 and many more), the public reaction was not based on the WHO facts but rather on the perceived reality in the online mass media. While we could not find any strong correlation between the media reporting and the WHO data on COVID-19, we found moderate and strong correlations 
between the media reporting and the Google Trends. The findings hold for all countries (Compare Table 2).

France was the only country where the media reporting was slightly more in line with the actual WHO reporting.

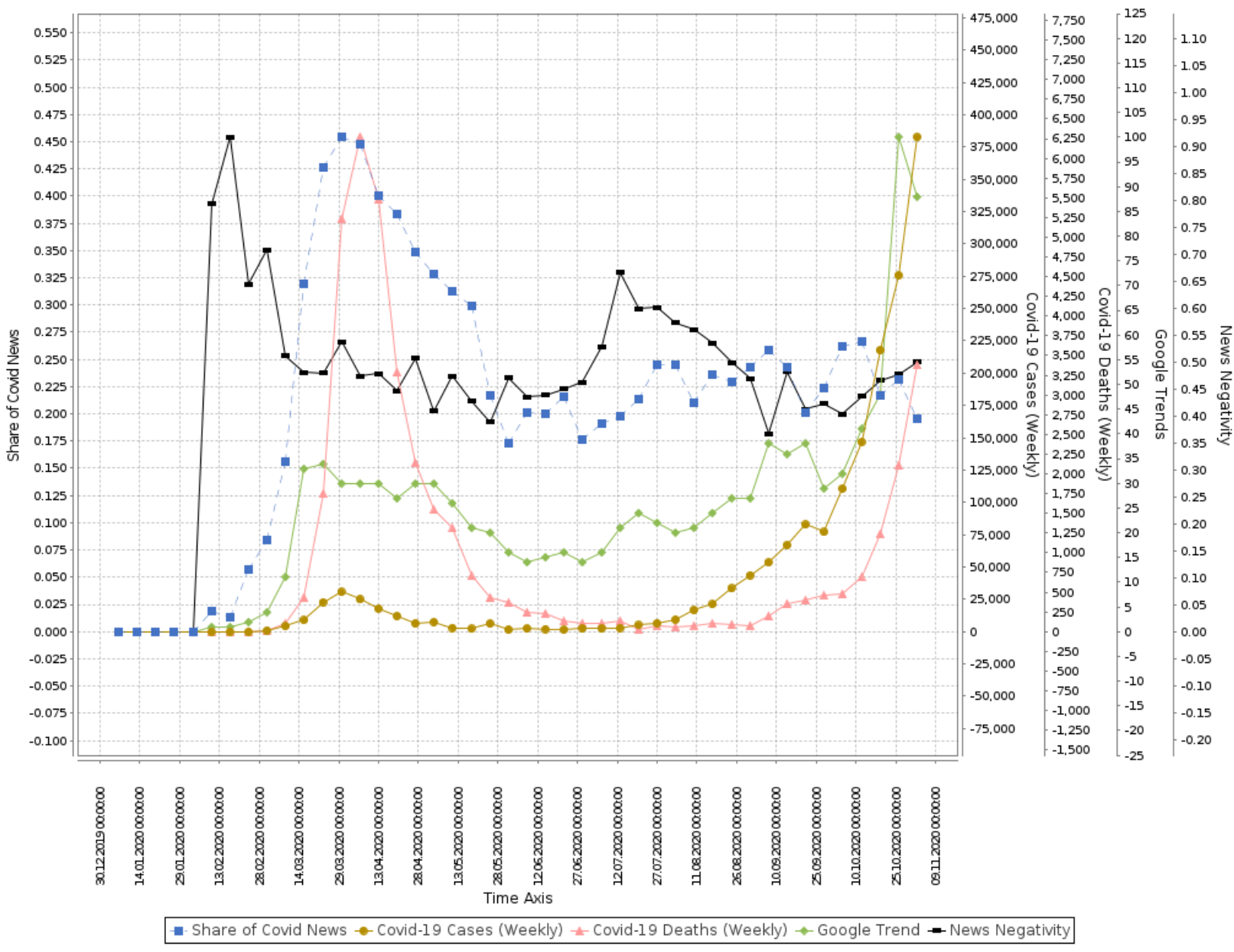

\section{Figure 4. Covid-19 Reporting in Relation to Covid-19 Data and Google Trends for France}

Figure 4 presents an overview of the correlations in France. In France, reporting after the first wave of the pandemic decreased strongly in line with the COVID-19 cases and deaths.

Table 2 shows the relations between the media reporting, the WHO data on COVID-19 cases and deaths and the Google Trends searches on COVID-19-related topics, for all countries. The table outlines that the described characteristics of an Infodemic also hold for all other countries under observation and there are barely significant correlations between the media reporting and the COVID-19 data from the WHO, but that the reactions on Google Trends follow the media reporting. 
Table 2. Correlation among Coverage, Negativity, COVID-19 Cases, COVID-19 Deaths and

\begin{tabular}{|c|c|c|c|c|c|c|}
\hline CH & 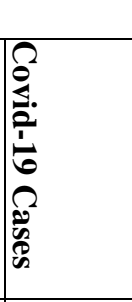 & & 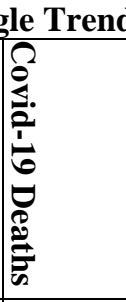 & & 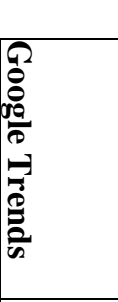 & \\
\hline Covid-19 Coverage & 0.0222 & 0.1669 & 0.2702 & $<0.01$ & 0.7759 & $<0.01$ \\
\hline Covid-19 Negativity & 0.0297 & 0.1353 & 0.0172 & 0.6058 & 0.0167 & 0.1951 \\
\hline \multicolumn{7}{|l|}{ DE } \\
\hline Covid-19 Coverage & 0.038 & 0.1077 & 0.2656 & $<0.01$ & 0.5695 & $<0.01$ \\
\hline Covid-19 Negativity & 0.0247 & 0.1556 & 0.0446 & 0.0901 & 0.3646 & $<0.01$ \\
\hline \multicolumn{7}{|l|}{ ES } \\
\hline Covid-19 Coverage & 0.1339 & $<0.01$ & 0.237 & $<0.01$ & 0.6381 & $<0.01$ \\
\hline Covid-19 Negativity & 0.0237 & 0.9516 & 0.0238 & 0.9942 & 0.002 & 0.3451 \\
\hline \multicolumn{7}{|l|}{ FR } \\
\hline Covid-19 Coverage & 0.0121 & 0.4896 & 0.4304 & $<0.01$ & 0.6897 & $<0.01$ \\
\hline Covid-19 Negativity & 0.016 & 0.5739 & 0.0042 & 0.3710 & 0.2015 & 0.0013 \\
\hline \multicolumn{7}{|l|}{ RO } \\
\hline Covid-19 Coverage & 0.0096 & 0.4471 & 0.0406 & 0.1004 & 0.7122 & $<0.01$ \\
\hline Covid-19 Negativity & 0.0056 & 0.3891 & 0.0365 & 0.1123 & 0.2812 & $<0.01$ \\
\hline \multicolumn{7}{|l|}{ UK } \\
\hline Covid-19 Coverage & 0.0986 & 0.0214 & 0.2967 & $<0.01$ & 0.6804 & $<0.01$ \\
\hline Covid-19 Negativity & 0.0246 & 0.1558 & 0.0197 & 0.6851 & 0.0584 & 0.0622 \\
\hline
\end{tabular}

Source: this study

Figure 5 shows a comparison of the media coverages in all countries. While the media coverage of all COVID-19 news after the first wave had larger fluctuations in France, Romania and Switzerland, it remained almost flat, on a high level in Germany, Spain and the UK. Still, the correlations between all countries are strong. 


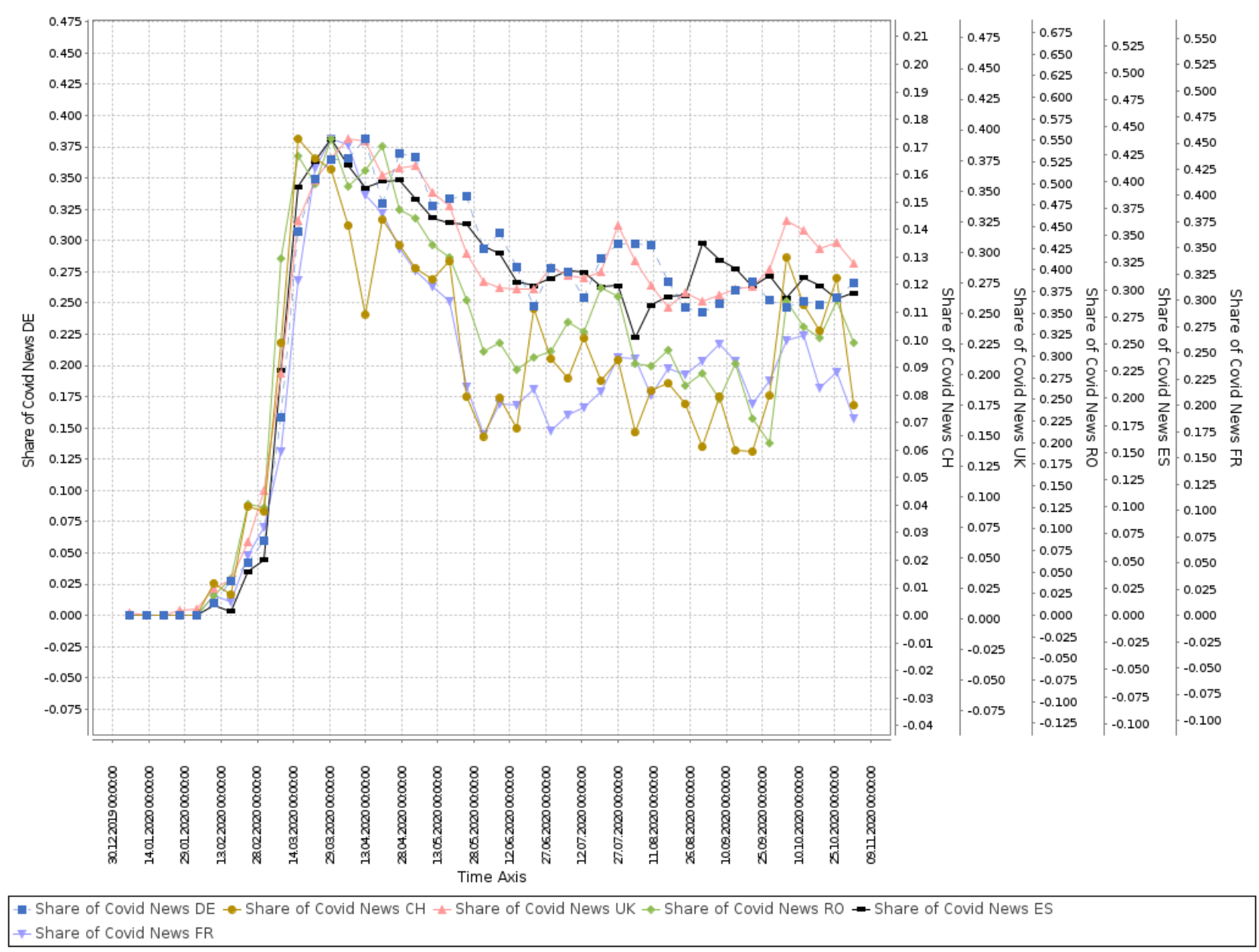

Figure 5. The Development of the Share of Covid-19 News

The figure shows that the media reporting in all countries under observation remained very high also during the summer months when the COVID-19 cases and deaths strongly decreased. The large share of COVID-19-related news on all news, which is of unprecedented dimensions, is remarkable.

Table 3 summarizes the share of COVID-19-related news on all news. This share is similar in a range between $23.3 \%$ for Germany and $30.13 \%$ for Romania, the only big exception is Switzerland with $8.3 \%$. While $8.3 \%$ for Switzerland might seem low, it is still a tremendous amount of news compared to all other topics that were of interest to the media in the last decade. These large shares of COVID-19 news and the large number of 202,608 COVID-19 news items in the sample underline the idea of overreporting and Infodemic.

Table 3. Share of COVID-19 News on all News

\begin{tabular}{|l|l|l|l|l|l|l|}
\hline & CH & DE & ES & FR & RO & UK \\
\hline $\begin{array}{l}\text { Average Share of Covid-19 } \\
\text { News }\end{array}$ & 0.0835 & 0.2333 & 0.2650 & 0.2051 & 0.3013 & 0.2453 \\
\hline
\end{tabular}

Figure 6 shows a comparison of the COVID-19 negativities of all news. The negativity for all countries (except France) is constant or slightly decreasing over time, but the levels of negativity are very different across countries. 

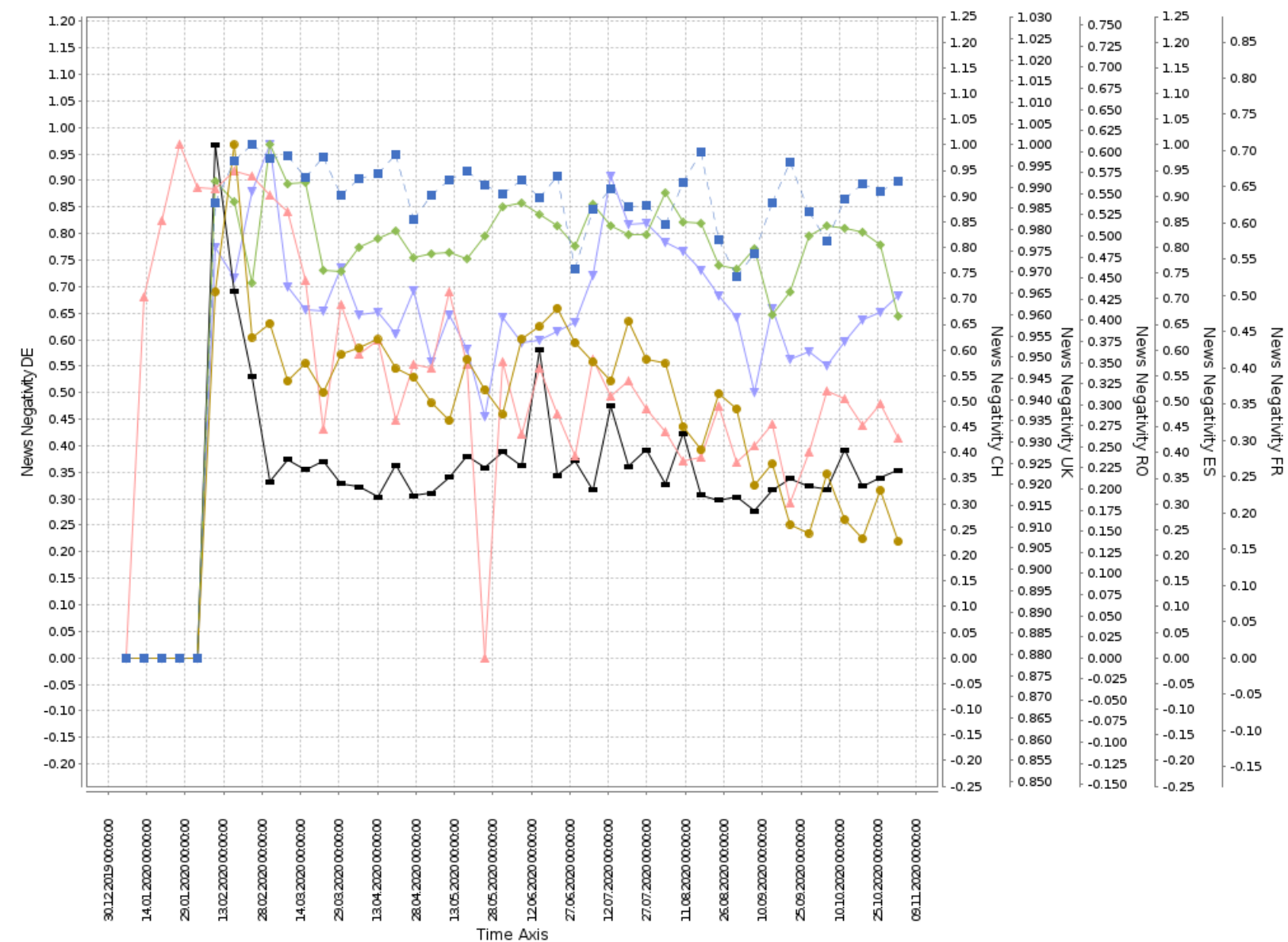

- News Negativity DE $\rightarrow$ - News Negativity $\mathrm{CH} \rightarrow$ News Negativity UK $\rightarrow$-News Negativity RO - - News Negativity ES $\rightarrow$ News Negativity FR

Figure 6. The Development of the Negativity of Covid-19 News

Table 4 shows the average negativities of the countries during the pandemic. While the negativity in Germany and the UK is very high with $87.9 \%$ and $94.7 \%$ of the news, respectively, the negativity in France, Romania, Spain and Switzerland is between $40.3 \%$ (for Spain) and 53.8\% (for Switzerland).

Table 4. Negativity of COVID-19 News

\begin{tabular}{|l|l|l|l|l|l|l|}
\hline & CH & DE & ES & FR & RO & UK \\
\hline $\begin{array}{l}\text { Average Negativity on } \\
\text { Covid-19 }\end{array}$ & 0.5375 & 0.8796 & 0.4026 & 0.5109 & 0.5059 & 0.9468 \\
\hline
\end{tabular}

Source: this study

Put in a nutshell, the results show that neither the media coverage nor the negativity reacted adequately to the WHO data on COVID-19 cases and deaths. Unfortunately, the empirical premise on which we based our study could not be confirmed from the data. Lower numbers of COVID-19 cases and deaths led neither to a lower media coverage nor to a lower negativity in the media. This clearly shows that the media did barely spread hope but rather continued to report with maximum intensity and maximum negativity. While negative news about the economy such as debts, wars and riots usually follow the facts (Förschler and Alfano, 2017; Shapiro, Sudhof and Wilson, 2017; Starosta, Budz and Krutwig, 2019b), the same cannot be confirmed in the case of the COVID-19 pandemic - a clear indicator for an Infodemic. Even more, in the case of this Infodemic, our study advocates that people reacted 
to the media and the perceived reality rather than to the facts. Further, the data outlined that in general media reporting during the COVID-19 pandemic was very similar among the countries under observation. Only rare outliers could be found as the slightly stronger fluctuations in the French media reporting and the much lower (but still incomparably high) media coverage in Switzerland.

\section{Discussion and Implications}

Media reporting on the COVID-19 pandemic has several demographic, economic, social, environmental, and political implications of which only a few could be captured and discussed in this section.

The first and most direct implication is that the headlines that are used for COVID-19 cannot be used for anything else. Thus, since March 2020 most other topics and headlines have disappeared from the European media and probably worldwide. A news coverage of $20 \%$ to $30 \%$ on COVID-19-related topics mutes almost all other issues and important news. Some topics might indeed have disappeared due to the worldwide pandemic but other topics are surely suppressed or lost because of the COVID-19 media reporting. Schattschneider (1975) states, "the definition of the alternatives [the agenda] is the supreme instrument of power". Moreover, ideas expressed in Tan and Weaver (2007), Birkland (2018), and Sciarini and Tresch (2019) prove to be true and on the political agenda in many European countries COVID-19 takes a large chunk of the administrative and parliamentary discussions. Thus, it is likely that the COVID-19 measures are not solely driven by the data of researchers and science but by the agenda of the online media as well.

Another problem is that the excessive media reporting on COVID-19 is even counterproductive for the message that should be delivered by the media. Since March, the media always screamed with maximum intensity, so not much of the ardour was left to expand the coverage and negativity during the start of the second wave of the COVID-19 pandemic. Thus, the appeals of the governments to pay attention to the measures or even new measures could hardly be communicated by the media, as there was no room for more intensive reporting and a crucial weapon of communication became ineffective.

The results are already visible in many countries as many people no longer take the measures seriously and do not follow them or interpret them to their advantage. Teralytics (2020) showed that mobility in Germany did barely react to the measures and the authorities' appeals during the second wave of the pandemic. According to data based on mobile phone providers, mobility did not decrease at all and was as high as prior to the pandemic. This was very different in Germany during the start of the pandemic in March 2020 when mobility decreased drastically. According to ETH et al. (2020) the same holds for Switzerland. While during the first wave of the pandemic mobility strongly decreased, the second wave only barely affected it, despite the country reporting of a higher number of COVID-19 cases and deaths. An Ifop (2020) survey shows that also in France the measures are only accepted and obeyed by $43 \%$ of the investigated population. According to their survey, $57 \%$ of the respondents have already bypassed the curfew and met partners, friends and family.

Finally, yet importantly, such a level of negativity in the news, as Aslam et al. (2020) argued in their study, might have important implications for 'emotional well-being and economic perspective'.

\section{Concluding Remarks}

Our study outlines how a COVID-19 Infodemic spread across the countries of observation, respectively: France, Germany, Romania, Spain, Switzerland, and the UK. This Infodemic is to the detriment of the masses. Many other important topics are muted in the media; the parliaments and authorities are in constant pressure to react to the developments in the 
media while their voices find their ways less and less to the citizens, making it very difficult to highlight the measures or enforce new measures. It was apparent that this Infodemic during the summer of 2020, with smaller numbers of COVID-19 cases and deaths, proved counterproductive to control the second wave. We hope that the transparency that this work has created will raise awareness not only among the media but also among citizens, policymakers, governments and administrations of the importance of balanced reporting and adequate media coverage. This will help in the future to carry messages to this effect, highlighting the negative impacts of distorted media coverage. Someone who is always screaming will no longer be paid attention to after some time as people get accustomed to the noisy situation. 


\section{References}

Agarwal, S., Kumar, S., \& Goel U. (2019). Stock market response to information diffusion through internet sources: A literature review. International Journal of Information Management, 45, 118-131. https://doi.org/10.1016/j.ijinfomgt.2018.11.002

Aslam, F., Awan, T.M., Syed, J.H., Kashif, A., \& Parveen, M. (2020). Sentiments and emotions evoked by news headlines of coronavirus disease (COVID-19) outbreak. Humanities and Social Sciences Communications. https://www.nature.com/articles/s41599-020-0523-3

Barkur, G., Vibha, \& Kamath, G.B. (2020). Sentiment analysis of nationwide lockdown due to COVID 19 outbreak: Evidence from India. Asian J Psychiatr. DOI: 10.1016/j.ajp.2020.102089

Birkland, T.A. (2017). Agenda Setting in Public Policy. Handbook of Public Policy Analysis Theory, Politics, and Methods. Routledge. https://doi.org/10.4324/9781315093192

Cinelli, M., Quattrociocchi, W., Galeazzi, A., Valensise, C.M., Brugnoli, E., Schmidt A.L., Zola, P., Zollo, F, \& Scala, A. (2020). The COVID-19 social media infodemic. Scientific Reports. https://www.nature.com/articles/s41598-020-73510-5

Buckman, S.R., Shapiro, A.H., Sudhof, M., \& Wilson, D.J. (2020, April 6). News Sentiment in the Time of COVID-19. FRBSF Economic Letter. https://www.frbsf.org/economicresearch/files/el2020-08.pdf

Chen, H., Huang, X., \& Li, Z. (2020). A content analysis of Chinese news coverage on COVID-19 and tourism. Current Issues in Tourism. DOI: 10.1080/13683500.2020.1763269.

ETH, IVT, WWZ, \& University of Basel. (2020, October 26). MOBIS-COVID19/26 Results as of 26/10/2020 (Second Wave). https://ivtmobis.ethz.ch/mobis/covid19/en, https://www.srf.ch/news/schweiz/mobilitaet-trotz-coronavirus-so-rege-ist-diebevoelkerung-aktuell-unterwegs

Förschler, F., \& Alfano, S. (2017). Reading between the lines: The effect of language sentiment on economic indicators. In S. Feuerriegel, \& D. Neumann, (Eds.), Enterprise Applications, Markets and Services in the Finance Industry (pp. 89-104). Springer Press.

Gills, B. (2020). Deep restoration: From the great implosion to the great awakening. Globalizations, 17(4), 577-579.

Google Trends (2020). Covid Search Terms. https://trends.google.com

Gopinath, G. (2020, April 14). The Great Lockdown: Worst Economic Downturn Since the Great Depression. IMF Blog. https://blogs.imf.org/2020/04/14/the-great-lockdownworst-economic-downturn-since-the-great-depression/

International Monetary Fund. (2020, June). World Economic Outlook. https://www.imf.org/en/Publications/WEO/Issues/2020/06/24/WEO

Guo, L. (2019). Media agenda diversity and intermedia agenda setting in a controlled media environment: A computational analysis of China's online news. Journalism Studies, 20(16), 2460-2477. https://doi.org/10.1080/1461670X.2019.1601029

Ifop (2020, November 12). Les Français et le reconfinement: entre dépression et transgression. https://www.ifop.com/publication/les-francais-et-le-reconfinement-entredepression-et-transgression/?utm_source=rss\&utm_medium=rss\&utm_campaign=lesfrancais-et-le-reconfinement-entre-depression-et-transgression

Imran, A.S., Daudpota, S.M., Kastrati, Z., \& Batra, R. (2020). Cross-cultural polarity and emotion detection using sentiment analysis and deep learning on COVID-19 related Tweets. IEEE Access, 8, 181074-181090. https://ieeexplore.ieee.org/abstract/document/9207881

Kruspe, A., Häberle, M., Kuhn, I., \& Zhu, X.X. (2020). Cross-language sentiment analysis of European Twitter messages during the COVID-19 pandemic. Proceedings of the 1st 
Workshop on NLP for COVID-19 at ACL 2020. Online.

https://www.aclweb.org/anthology/2020.nlpcovid19-acl.14/

Martin, C.A., Torres, J.M., Aguilar, R.M., \& Diaz, S. (2018). Using deep learning to predict sentiments: Case study in tourism. Complexity, 2018, Article ID 7408431. https://doi.org/10.1155/2018/7408431

Maurer, M. (2017). Agenda-setting (2nd ed). Nomos. https://doi.org/10.5771/9783845283043

McCombs, M.E., \& Shaw, D.L. (1972). The agenda-setting function of mass media. The Public Opinion Quarterly, 36(2), 176-187.

Mikolov, T., Chen, K., Corrado, G., \& Dean, J. (2013). Efficient estimation of word representations in vector space. https://arxiv.org/abs/1301.3781

Onete, B.C., Starosta, K., Budz, S., \& Krutwig, M. (2018). Estimating perceptions of consumers using AI: A case study for German and UK travelers. BASIQ International Conference "New trends in sustainable business and consumption" 2018 Conference Proceedings, Germany, 181-188. https://conference.ase.ro/pdf/BASIQ\%202018$\% 20$ Volume.pdf

Patterson, J., \& Gibson, A. (2017). Deep Learning (1st ed.). O'Reilly Media.

Schattschneider, E.E. (1975). The semisovereign people: A realist's view of democracy in America. Dryden Press.

Scheufele, D.A., \& Tewksbury, D. (2007). Framing, agenda setting, and priming: the evolution of three media effects models. Journal of Communication, 57(1), 9-20. https://doi.org/10.1111/j.1460-2466.2006.00326_5.x

Schwitzer, G., Mudur, G., Henry, D., Wilson, A., Goozner, M., Simbra, M., Sweet, M., \& Baverstock, K. A. (2005). What are the roles and responsibilities of the media in disseminating health information? PLoS Medicine, 2(8), e321. https://doi.org/10.1371/journal.pmed.0020215

Sciarini, P., \& Tresch, A. (2019). The political agenda-setting power of the media: The Europeanization nexus. Journal of European Public Policy, 26(5), 734-751. https://doi.org/10.1080/13501763.2018.1458890

Shapiro, A., Sudhof, M., \& Wilson, D.J. (2017). Measuring news sentiment, Federal Reserve Bank of San Francisco. Working Paper 2017-01. http://www.frbsf.org/economic-research/publications/working-papers/wp2017-01.pdf

Starosta, K., Onete, C.B., Budz, S., \& Krutwig, M. (2019). Differences in travelers' perceptions of popular tourist destinations estimated by an LSTM neural network: A comparison between the UK and Germany. Tourism: An International Interdisciplinary Journal, 67(4), 405-422.

Starosta, K., Budz, S., \& Krutwig, M. C. (2019a). Low media coverage drives a biased picture in the news (And the contrary). Proceedings of the 6th European Conference on Social Media, ECSM 2019, UK, p. 351.

Starosta, K., Budz, S., \& Krutwig, M. (2019b). The impact of German-speaking online media on tourist arrivals in popular tourist destinations for Europeans. Applied Economics, 51(14), 1558-1573.

Tan, Y., \& Weaver, D.H. (2007). Agenda-setting effects among the media, the public, and congress, 1946-2004. Journalism \& Mass Communication Quarterly, 84(4), 729-744. https://doi.org/10.1177/107769900708400405.

Teralytics, 2020. COVID-19 resource centre - What mobility tells us about recovery in Europe. https://www.teralytics.net/post-pandemic-recovery-center/?selected_data=de , https://interaktiv.tagesspiegel.de/lab/corona-fast-so-viele-reisen-wie-vor-der-pandemie/

WHO, 2020. WHO coronavirus disease (COVID-19) dashboard. https://covid19.who.int/

Zhang, L., Wang, S., \& Liu, B. (2018). Deep learning for sentiment analysis: A survey. IREs Data Mining Knowledge Discovery, 8(4). https://doi.org/10.1002/widm.1253 
Zhou, J., Yang, S., Xiao, C., \& Chen, F. (2020). Examination of community sentiment dynamics due to covid-19 pandemic: a case study from Australia. https://arxiv.org/abs/2006.12185

Zhu, B., Zheng, X., Liu, H., Li, J., \& Wang, P. (2020). Analysis of spatiotemporal characteristics of big data on social media sentiment with COVID-19 epidemic topics. Chaos, Solitons \& Fractals, 140. https://doi.org/10.1016/j.chaos.2020.110123 